\title{
Automatic Border Detection of the Left Ventricle in Parasternal Short Axis View of Echocardiogram
}

\author{
G.N.Balaji \\ Ph.D. Research Scholar \\ Department of Computer Science and Engineering \\ Annamalai University, Annamalai Nagar
}

\author{
T.S.Subashini, Ph.D \\ Associate professor \\ Department of Computer Science and Engineering \\ Annamalai University, Annamalai Nagar
}

\begin{abstract}
Echocardiogram is one of the easiest ad widely employed methods that uses ultrasound to evaluate heart muscle, heart valves, and risk for heart disease. Heart failure (HF) can result from any structural or functional cardiac disorder that impairs the ability of the ventricle to fill with or eject blood. Echocardiography represents "the gold standard" in the assessment of left ventricle LV systolic and diastolic dysfunction. Left ventricular dimensions, volumes and wall thicknesses are echocardiographic measurements that are widely used in clinical practice and research. To obtain accurate linear measurements from the echocardiography accurate segmentation of the LV is essential. This paper proposes a method to segment the left ventricular border automatically on the 3-dimensional $(2 \mathrm{D}+\mathrm{t})$ echocardiogram, where ' $t$ ' is the time. The 2D image is obtained by extracting the frames from the video of echocardiogram which is further processed to detect the edges of the left ventricle and finally the edge detected frames are converted back into video which will help the cardiologist to visualize the left ventricle in motion. The obtained results are efficient and can be utilized for the purpose of detecting various medical parameters.
\end{abstract}

\section{Keywords}

Echocardiogram, Left ventricular, automatic detection, segmentation.

\section{INTRODUCTION}

Echocardiography images the heart using standard 2D, 3D and Doppler ultrasound [1]. The human heart is an organ that provides continuous blood circulation to the entire body through the cardiac cycle and is one of the most vital organs in the human body [2]. The heart is divided into four main chambers: the two upper chambers are called the left and right atria and two lower chambers are called the right and left ventricles [3]. The left ventricle [LV] receives oxygenated blood from the left atrium via the mitral valve, and pumps it into the aorta via the aortic valve [4]. For healthy functioning of the heart the LV should relax very rapidly after each contraction so as to fill rapidly with oxygenated blood owing from the lung veins. The echocardiogram allows doctors to diagnose, evaluate, and monitor heart related problems. An echocardiogram is a test that uses sound waves to create a moving picture of the heart $[5,6]$. The picture is much more detailed than a plain $\mathrm{x}$-ray image and involves no radiation exposure. An echocardiogram allows doctors to see the heart beating, and to see the heart valves and other structures of the heart. Clinical parameters such as ejection fraction (EF), left ventricle myocardium mass (MM), and stroke volume (SV) are required by the cardiologist for accurate diagnosis of heart related problems. Calculations of these parameters depend upon accurate delineation of endocardial and epicardial contours of the left ventricle (LV). Manual delineation is time consuming and tedious and has high inter-observer variability. Thus, fully automatic LV segmentation in echocardiogram is desirable. The automatic segmentation of the LV in echocardiogram typically faces four challenges: 1)the overlap between the intensity distributions within the cardiac regions; 2)the lack of edge information. 3) The shape variability of the endocardial and epicardial contours and 4) the inter-subject variability of these factors. In this paper it is proposed to automatically segment the LV in parasternal short-axis view of the echocardiogram video without any manual intervention.

\section{RELATED WORK}

Accurate LV segmentation is quite difficult due to the presence of noise and due to the low contrast of echocardiograph image. Hence preprocessing of echocardiograph images is very important before the actual segmentation of LV. The work in [7] presented a waveletbased thresholding scheme for noise suppression in ultrasound images. In [8] the image is filtered by convolving with a $3 \times 3$ Gaussian low pass filter followed by thresholding and to eliminate the noise morphological dilation and erosion have been applied. Adaptive weighted median filter (AWMF) for reducing speckle noise in ultrasound images is presented in [9] which is based on the weighted median. By adjusting the weight coefficients and consequently the smoothing characteristics of the filter according to the local statistics around each point of the image, it is possible to suppress noise while edges and other important features are preserved. The work in [10] combines image processing techniques with radial search to detect the left ventricular borders from echocardiograph images. The fuzzy reasoning employed in [11] defines edges by local image characteristics computed based on local statistics of the image. In this work to reduce the noise and to enhance the contrast of the image high boost filtering followed by LoG filter is carried out. The literature shows different methods for segmentation of LV in short axis views. The work in [12] uses the gray level information along with user defined initial contours to extract the boundary in the images. The method in [13] implements a probabilistic deformable model considering the boundary as twodimensional deformable object using maximum posteriori estimate. The work in [14] reports an interesting approach to detect LV boundary of short axis echocardiographic 
sequences using a multiple active contour model which is an extension to the original model proposed in [15]. A Hough transform technique is used in [16] to find an initial approximation of the object boundary at the first frame of the sequence then, an active-contour model is used in a coarse-tofine framework, for the estimation of a noisy LV (Left Ventricle) boundary. A texture segmentation algorithm based on the multi-channel filtering theory, is presented in [17]. The work in [18] presents a technique that incorporates the information on pixel velocities (optical flow) into the estimate of initial contour to enable tracking of fast-moving objects. Watershed transform and morphological operation to locate the region containing the LV which then performs snake deformation with a multi-scale directional edge map for the detection of the endocardial boundary of the LV is proposed in [19]. More recently a novel approach of combining the $\mathrm{K}$ Means clustering algorithm for segmentation and active contour model for boundary detection was proposed in [20]. In this work of mathematical morphology and connected component labeling are used to segment the LV in short axis view echocardiography.

\section{METHODOLOGY}

The block diagram of the proposed work is shown inFigure1.The Echocardiogram video is given as input to the system and is converted to frames which are then subjected to further processing.

Input: Echocardiogram Video

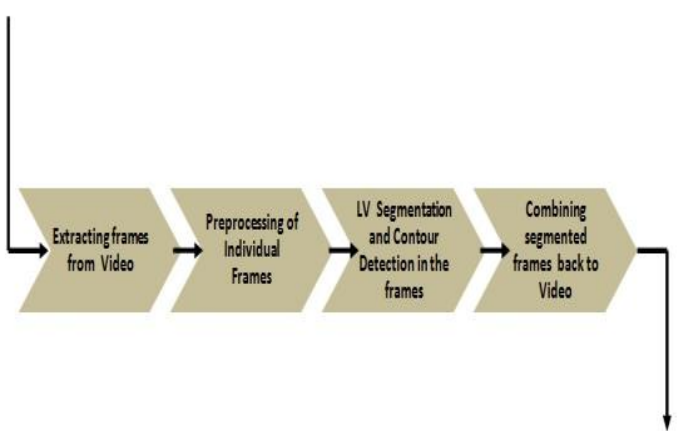

Output: Echocardiogram Video

with LV Highlighted

Figure 1: The proposed system.

The extracted frame is shown in Figure 2. The noise reduction and contrast enhancement operations are performed as a preprocessing step to highlight the region of interest namely the left ventricle. Preprocessing filters namely high boost filter and Laplacian of Gaussian (LoG) filters are applied to reduce noise and increase contrast in the individual frame extracted from video. It is often desirable to emphasize high frequency components representing the image details without eliminating low frequency components.

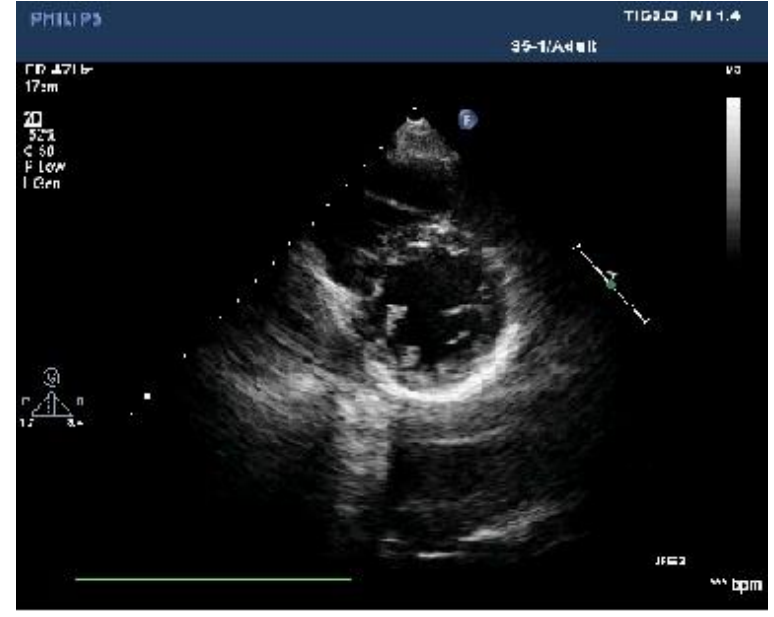

Figure 2: The extracted frame

In this work the high-boost filter is used to enhance high frequency component while still keeping the low frequency components.

Table 1: PSNR values of denoising filters

\begin{tabular}{|c|c|c|}
\hline $\begin{array}{c}\text { Denoising } \\
\text { method }\end{array}$ & PSNR & Reference \\
\hline $\begin{array}{l}\text { LP transform } \\
\text { based } \\
\text { despeckling } \\
\text { method }\end{array}$ & 28.44 & [22] \\
\hline $\begin{array}{c}\text { WT-L3-ST (bior } \\
6.8 \text { ) }\end{array}$ & 29.14 & [22] \\
\hline Wiener $(3 \times 3)$ & 32.14 & [22] \\
\hline High boost filter & 33.16 & proposed \\
\hline
\end{tabular}

Highpass $=$ original - lowpass

If original is multiplied by an amplification factor $\mathrm{A}$, a high boost image will result.

$$
\begin{aligned}
& \text { High boost }=(\mathrm{A}) *(\text { original })-\text { lowpass } \\
& \begin{array}{r}
(\mathrm{A}-1) *(\text { original })-\text { lowpass } \\
\quad+(\text { original }- \text { lowpass })
\end{array} \\
& =(A-1) *(\text { original })-\text { lowpass }+ \text { highpass }
\end{aligned}
$$

The high boost filtered image is shown in Figure 3. Laplacian filters are second order derivative filters used to find areas of rapid change (edges) in images. Since second order derivative filters are very sensitive to noise, it is common to smooth the image (e.g., using a Gaussian filter) before applying the Laplacian. This two-step process is calling the Laplacian of Gaussian (LoG) operation. 


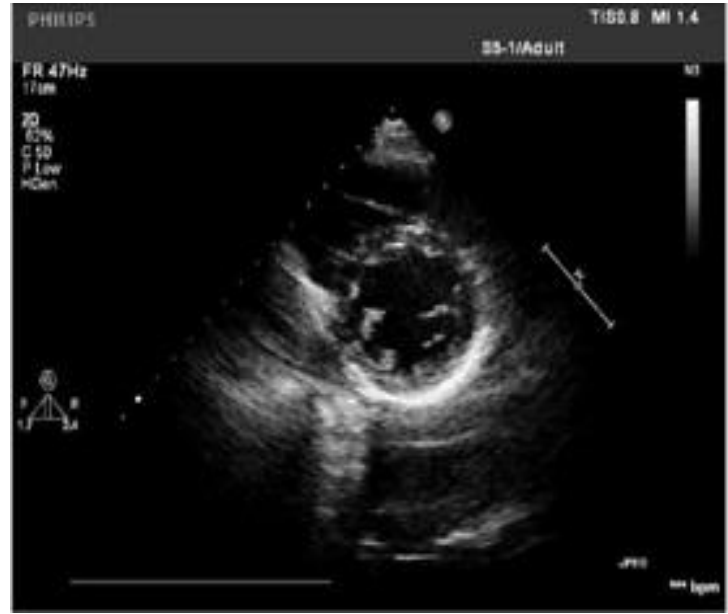

Figure 3: The high boost filtered image

Step1:

First the image is smoothened using Gaussian operator

$$
\mathrm{h}(\mathrm{x}, \mathrm{y})=\exp \left(\frac{-x^{2}-y^{2}}{2 \sigma^{2}}\right)
$$

Where $\sigma$ represents standard deviation,

Step2:

Then Laplacian operator is applied

$$
x^{2}+y^{2}=r^{2}
$$

Laplacian $\nabla^{2} h=\left(\frac{r^{2}-\sigma^{2}}{\sigma^{4}}\right) \exp \left(\frac{-r^{2}}{2 \sigma^{2}}\right)$

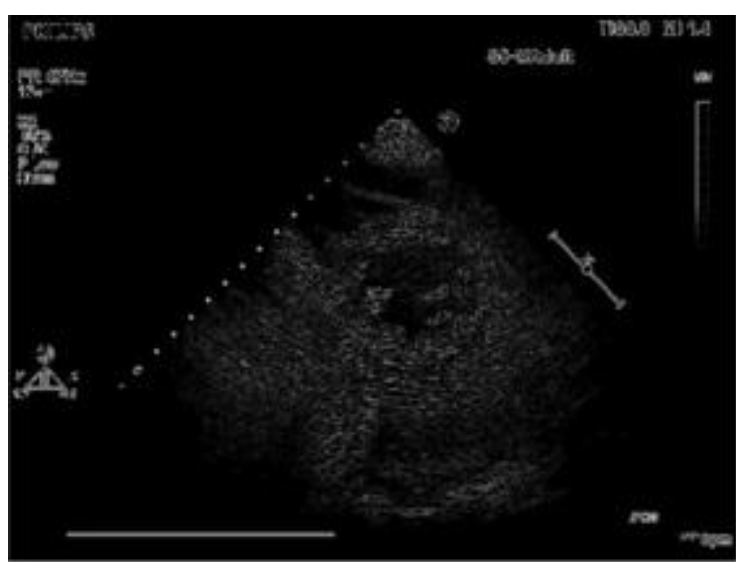

Figure 4: LoG applied image.

Where the image is basically uniform, the LoG filter will give a response of zero. Wherever a change occurs, the LoG will give a positive response on the darker side and a negative response on the lighter side of the edge. Figure 4. shows the LoG operated image. Table 1 shows a comparison of PSNR values for various filters used in the literature. It can be seen that high boost filter gives better PSNR when compared to other filters. The next step is segmentation of the LV. Figure 5 shows the steps included in segmentation process.

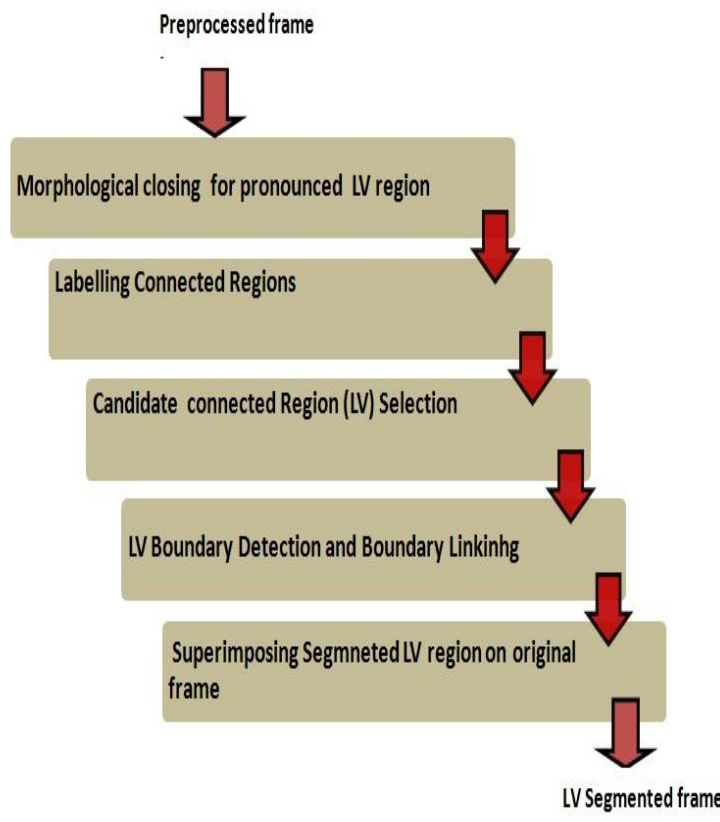

Figure 5: steps of segmentation process.

As the initial step morphological closing is performed in order to obtain the cardiac cavity as shown in Figure 6 (a). Next connected-component labeling is applied to obtain the connected regions as shown in Figure 6 (b)

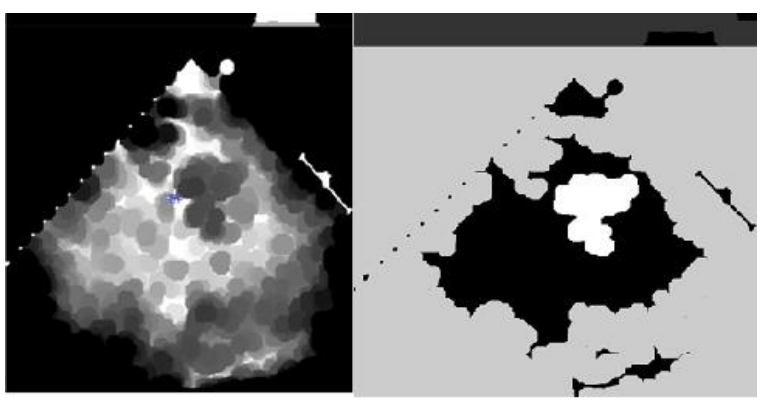

(a)

(b)

Figure 6: (a) Morphological closing, Fig (b) ConnectedComponent labeling.

Connected-component labeling works by scanning an image, pixel by pixel (from top to bottom and left to right) in order to identify connected pixel regions, i.e. regions of adjacent pixels which share the same set of intensity values $\mathrm{V}$ [21]. The candidate region that is the $\mathrm{LV}$ is obtained by discarding those components less than 4000 pixels and greater than 65000 pixels. These values were found empirically and this works fine for all short axis echocardiogram images. The detected LV region is shown in Figure 7. Boundary detection and boundary linking is performed after LV detection as shown in Figure 7(b). Next step is superimposing the boundary detected image with original image. In the final step boundary detected individual frames are transformed into echocardiogram video as shown in Figure 8. The reconstructed video shows the left ventricle highlighted and this helps the cardiologist to visualize the left ventricular motion during a cardiac cycle. 
a)
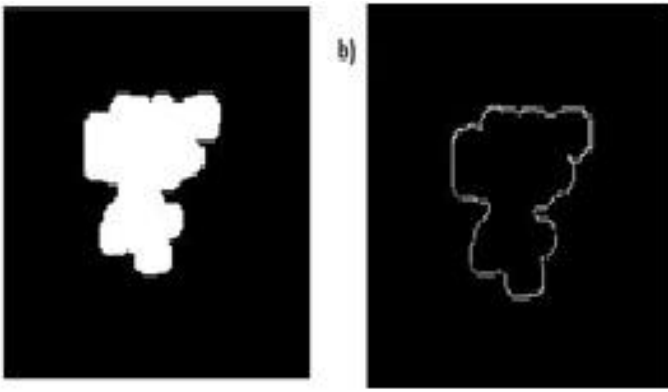

Figure 7: (a) segmented LV, Fig. (b) After boundary linking.

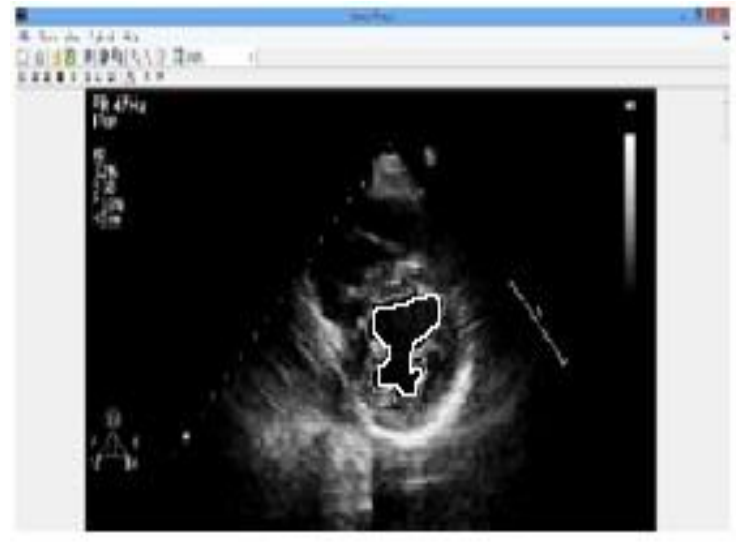

Figure 8: Frames reconstructed back to video

\section{CONCLUSION}

In this paper a method for automatic border detection in left ventricle was proposed using the classic techniques of mathematical morphology and connected component labeling. The left ventricular motion over a complete cardiac cycle was provided by the proposed algorithm. The accuracy of the proposed method was ascertained by the cardiologist. When compared to manually segmented LV by the cardiologist the proposed algorithm is accurate in automatically segmenting the left ventricle in parasternal short axis view echocardiography. The variations observed in manual border delineations were eliminated by the proposed method. Hence the proposed method can be very effectively used in LV segmentation.

\section{ACKNOWLEDGMENT}

The authors would like to thank Dr.B.Neelakandan, M.S., Mch. D.N.B., FRCS. And Dr.V.Ravi, DM. for their invaluable contribution.

\section{REFERENCES}

[1] Frazin L, Talano JV, Stephanides L, Loeb HS, Kopel L, Gunnar RM.Esophageal echocardiography. Circulation.

[2] Hisanaga K, Hisanaga A, Nagata K, Ichie Y. Transesophageal crosssectional echocardiography. Am Heart J 1980.

[3] Schluter M, Henrath P. Transesophageal echocardiography: potential advantages and initial clinical results. Practical Cardiol.. 1983;9:149.
[4] Omoto, Ryozo, et al. "The development of real-time twodimensional Doppler echocardiography and its clinical significance in acquired valvular diseases. With special reference to the evaluation of valvular regurgitation." Japanese heart journal 25.3 (1984): 325-340.

[5] Strandness Jr, D. E., et al. "Transcutaneous directional flow detection: A preliminary report." American heart journal 78.1 (1969): 554-556.

[6] Baker DW, Rubenstein SA, Lorch GS. Pulsed Doppler echocardiography principles and applications. Am J Med.. 1977; 63:69-80.

[7] S.Sudha, G.R.Suresh and R.Sukanesh Speckle Noise Reduction in Ultrasound Images by Wavelet Thresholding based on Weighted Variance, International Journal of Computer Theory and Engineering, Vol. 1, No.1, April 2009.

[8] A. Mishra, P. K. Dutta, and M. K. Ghosh, A GA based approach for boundary detection of left ventricle with echocardiographic image sequences. Image Vis. Compute., vol. 21, pp. 967-976, 2003.

[9] T. Loupas, W. N. Mcdicken, and P. L. Allan. An Adaptive Weighted Median Filter for Speckle Suppression in Medical Ultrasonic Images OO98-4094/89/010O012901.01989 IEEE.

[10] Saulo Guerreiro Lacerda, Adson F. da Rocha, Daniel F. Vasconcelos,Joo L. A. de Carvalho, Iwens G. Sene Jr. and Juliana F. Camapum Left Ventricle Segmentation in Echocardiography Using a Radial-Search-Based Image Processing Algorithm. IEEE 2008.

[11] Sheila Chan, Gopalakrishnan Sainarayanan, Fuzzy-based boundary enhancement for echocardiogram using local image characteristics. Malaysian Journal of Computer Science, Vol. 19(2), 2006.

[12] Chu, C. H., Delp, E. J., and Buda, A. J. (1988). Detecting left ventricular endocardial and epicardial boundaries by digital two dimensional echocardiography. IEEE Transactions on Medical Imaging.

[13] Staib, L. H., and Duncan, J. S. (1992). Boundary finding with parametrically deformable models. IEEE Transactions on Pattern Analys is and Machine Intelligence.

[14] Chalana, V., Linker, D. T., Haynor, D. R., and Kim, Y. (1996).A multiple active contour model for cardiac boundary detection one echocardiographic sequences.IEEE Transactions on Medical Imaging,15, 290298.

[15] Kass M., Witkin A., and Terzopoulos D. (1988). Snakes: Active contour models. International Journal of Computer Vision, 1, 321331.

[16] Antonio Fernandez-Caballero, Jose M. Vega-Riesco. Determining heart parameters through left ventricular automatic segmentation for heart disease diagnosis. Expert Systems with Applications 36 (2009) 223422492007ElsevierLtd.All rights reserved doi:10.1016/j.eswa.2007.12.045.

[17] A Bosnjak, G Montilla, V Torrealba, Medical Images Segmentation using Gabor Filters applied to Echocardiographic Images0276-6547/981998 IEEE. 
[18] IvanaMikic,* Slawomir Krucinski, and James D. Thomas, Segmentation and Tracking in Echocardiographic Sequences: Active Contours Guided by Optical Flow Estimates S 0278-0062(98)04883-6. 1998 IEEE.

[19] Jierong Cheng, Say Wei Foo, and Shankar M. Krishnan Watershed Presegmented Snake for Boundary Detection and Tracking of Left ventricle in Echocardiographic Images IEEE transactions on informationtechnology in biomedicine, vol. 10, no. 2, April 2006.
[20] S. Nandagopalan, C. Dhanalakshmi, Dr. B. S. Adiga N. Deepak, Automatic Segmentation and Ventricular Border Detection of 2DEchocardiographic Images Combining K-Means Clustering and Active Contour Model. IEEE 2010.

[21] R.c.Gonzanez and R.e woods DIP, Pearson education Singapore, 2002.

[22] Zhang F.,Koh L.M., Yoo Y.M. and Kim Y.,2007, Nonlinear diffusion in Laplacian pyramid domain for ultrasonic speckle reduction, IEEE Trans. on Medical Imaging. 